\title{
Numerical simulation of Faraday waves oscillated by two-frequency forcing
}

\author{
AUTHOR(S): \\ Takagi, Kentaro; Matsumoto, Takeshi
}

CITATION:

Takagi, Kentaro ...[et al]. Numerical simulation of Faraday waves oscillated by two-frequency forcing. Physics of Fluids 2015, 27(3): 032108.

\section{ISSUE DATE:}

2015-03-24

URL:

http://hdl.handle.net/2433/216144

\section{RIGHT:}

(c) 2015 AIP Publishing LLC. This article may be downloaded for personal use only. Any other use requires prior permission of the author and AIP Publishing. The following article may be found at

http://scitation.aip.org/content/aip/journal/pof2/27/3/10.1063/1.4915340 


\section{AIP $\mid$ Physics of Fluids}

\section{Numerical simulation of Faraday waves oscillated by two-frequency forcing}

Kentaro Takagi and Takeshi Matsumoto

Citation: Physics of Fluids (1994-present) 27, 032108 (2015); doi: 10.1063/1.4915340

View online: http://dx.doi.org/10.1063/1.4915340

View Table of Contents: http://scitation.aip.org/content/aip/journal/pof2/27/3?ver=pdfcov

Published by the AIP Publishing

\section{Articles you may be interested in}

Low-frequency vortex oscillation around slender bodies at high angles-of-attack

Phys. Fluids 26, 091701 (2014); 10.1063/1.4895599

Aspect ratio and radius ratio dependence of flow pattern driven by differential rotation of a cylindrical pool and a disk on the free surface

Phys. Fluids 25, 084101 (2013); 10.1063/1.4817179

Pattern formation in a reaction-diffusion-advection system with wave instability Chaos 22, 023112 (2012); 10.1063/1.4704809

Three-dimensional numerical study of natural convection in vertical cylinders partially heated from the side

Phys. Fluids 17, 124101 (2005); 10.1063/1.2141430

Large-eddy simulation of low frequency oscillations of the Dean vortices in turbulent pipe bend flows

Phys. Fluids 17, 035107 (2005); 10.1063/1.1852573

\section{Did your publisher get}

18 MILLION DOWNLOADS in 2014?

\section{AIP Publishing did.}

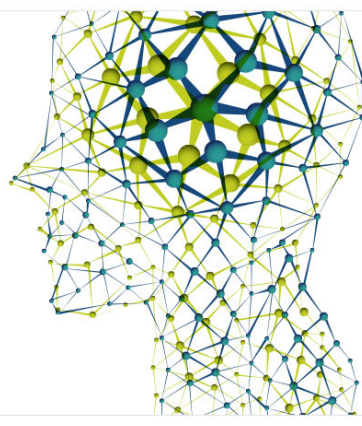




\title{
Numerical simulation of Faraday waves oscillated by two-frequency forcing
}

\author{
Kentaro Takagi ${ }^{\mathrm{a})}$ and Takeshi Matsumoto ${ }^{\mathrm{b}}$ \\ Division of Physics and Astronomy, Graduate School of Science, Kyoto University, \\ Kitashirakawa Oiwaketyo, Sakyoku, Kyoto 606-8502, Japan
}

(Received 19 September 2014; accepted 14 February 2015; published online 24 March 2015)

\begin{abstract}
We perform a numerical simulation of Faraday waves forced with two-frequency oscillations using a level-set method with Lagrangian-particle corrections (particle level-set method). After validating the simulation with the linear stability analysis, we show that square, hexagonal, and rhomboidal patterns are reproduced in agreement with the laboratory experiments [Arbell and Fineberg, "Two-mode rhomboidal states in driven surface waves," Phys. Rev. Lett. 84, 654-657 (2000) and "Temporally harmonic oscillons in Newtonian fluids," Phys. Rev. Lett. 85, 756-759 (2000)]. We also show that the particle level-set's high degree of conservation of volume is necessary in the simulations. The numerical results of the rhomboidal states are compared with weakly nonlinear analysis. Difficulty in simulating other patterns of the two-frequency forced Faraday waves is discussed. (C) 2015 AIP Publishing LLC. [http://dx.doi.org/10.1063/1.4915340]
\end{abstract}

\section{INTRODUCTION}

Faraday waves, ${ }^{1}$ known to exhibit various kinds of crystalline patterns in simple settings, have attracted many researchers for about two hundred years. Faraday waves are the surface waves between two superposed immiscible fluid layers subjected to a vertical vibration. Even recently astounding exotic phenomena continue to be found in laboratory experiments on Faraday waves. For example, in Faraday waves with a certain non-Newtonian fluid (shear-thickening fluid), the behavior of the interface is far beyond what one can imagine from the interface motion between air and water. ${ }^{2}$ In another surprising experiment, a droplet slightly submerged in a liquid substrate under a vertical oscillation is found to behave dynamically like a snake. ${ }^{3}$ To physically understand these phenomena, numerical simulations of them, which may not be possible now, are expected to play a decisive role.

As a first step to build such numerical methods, we here study numerically Faraday waves subjected to a two-frequency forcing in a Newtonian fluid. There are a number of experimental results with this forcing setting, ${ }^{4-13}$ where much richer variations of the selected patterns are found than in the single-frequency forced cases as listed below.

The study of two-frequency forced Faraday waves starts with the experiments by Edwards and Fauve $^{4,5}$ and Muller. ${ }^{6}$ The two-frequency forcing can be written as $A_{1} \cos \left(m \omega_{0} t\right)+A_{2} \cos \left(n \omega_{0} t+\phi\right)$ and characterized by the integers $m$ and $n$. Edwards and Fauve explored various ratios of the two frequencies such as $m: n=3: 5,4: 5,6: 7,4: 7$, and 8:9 and mainly investigated the ratio 4:5. They observed the quasi-pattern, which has a long-range orientational order but no spatial periodicity. On the other hand, the experiment by Muller is focused on the driving ratio of 1:2 and produces a triangular pattern.

In the linear regime of the two-frequency forced case, a bicritical point exists at which two normal modes with different wavenumber moduli become simultaneously unstable (for the single-frequency forced case, the bicritical point can be formed by tuning the frequency of the forcing for shallow layers ${ }^{14}$ ). The unstable modes then interact with each other nonlinearly. In the neighborhood of the

\footnotetext{
a)Electronic mail: kentaro@kyoryu.scphys.kyoto-u.ac.jp

b) Electronic mail: takeshi@kyoryu.scphys.kyoto-u.ac.jp
} 
bicritical point, many complex patterns are expected to be found. A number of experiments around the bicritical point were conducted by Kudrolli et al. ${ }^{7}$ Arbell and Fineberg,${ }^{8-11}$ and Epstein and Fineberg. ${ }^{12,13}$ Kudrolli et al. observed patterns that they named superlattice-1 and superlattice-2. Arbell and Fineberg and Epstein and Fineberg observed double hexagonal superlattice (DHS), subharmonic superlattice states (SSS), oscillon, two-mode superlattices (2MS), and $2 k$ rhomboidal states $(2 k \mathrm{R})$. Each pattern can be characterized by the number of excited (discrete) Fourier modes and by the nonlinear resonance among them.

To the best of our knowledge, numerical simulation of the two-frequency forced Faraday waves based on the Navier-Stokes equations solving the motion of both the top and bottom fluids is reported in this paper for the first time. However, such a simulation, not limited to the two-frequency forced case, requires treatment of the interface with the surface tension force. We therefore must employ one of the interface-tracking schemes such as the volume-of-fluid methods, the level-set methods, and the front-tracking methods (see, e.g., an advanced textbook ${ }^{15}$ ). In this study, we adopt the level-set method and investigate whether or not the simulation of two-frequency forced Faraday waves with the level-set method is consistent with the experimental results. The reason for adopting the level-set method will be described later.

The first numerical simulation of the single-frequency forced Faraday waves in three dimensions was performed by Périnet et al. ${ }^{16}$ who reproduced the square and hexagonal patterns in quantitative agreement with the laboratory experiment by Kityk et al. ${ }^{17}$ Périnet et al. ${ }^{16}$ used a fronttracking method. It is necessary, for example, in simulating oscillon or snake-like patterns, to allow for overturning and topological change of the interface. We hence believe that other interface-tracking schemes should be explored and tested for a wider class of the Faraday waves. Another numerical issue concerns the density difference between the top and the bottom fluids. In typical laboratory experiments, these are air and water at room temperature, meaning three orders of magnitude difference in the densities. To handle this large difference, it is known that a high quality solver for the pressure Poisson equation is needed regardless of the choice of interface-tracking scheme. ${ }^{15}$ We use a preconditioned BiCGSTAB.

On the theoretical front of the two-frequency forced Faraday waves, linear stability analysis and weakly nonlinear theory are available. Linear analysis was performed by Besson and Edwards, ${ }^{18}$ which is an extension of the single-frequency forced case. ${ }^{19}$ Their results ${ }^{18}$ agree with the experiments quantitatively. In the weakly nonlinear analysis, whose emphasis is on the pattern selection of the two-frequency Faraday waves, Silber et al., Tse et al., Porter et al., and Topaz et al. ${ }^{20-26}$ formulated an amplitude equation up to third order in amplitude by applying symmetry based arguments.

By analyzing the structure of the three-wave resonance, they succeeded in explaining many selected patterns qualitatively. Quantitative prediction of the pattern can be obtained if the amplitude equation of the two-frequency forced Faraday waves is derived from the Navier-Stokes equation with a realistic boundary condition. However this is a formidable task. A reduced hydrodynamic equation of the two-frequency Faraday waves was derived by Zhang and Viñals. ${ }^{27}$ From this reduced equation, the amplitude equations are derived and analyzed by assuming infinite depth and small viscosity. ${ }^{25-27}$ Weakly nonlinear analysis based on the Navier-Stokes equations with infinite depth was carried out by Skeldon and Guidoboni. ${ }^{28}$ This approach with realistic amplitude equations is successful in explaining many patterns observed in the two-frequency forced Faraday waves. Nevertheless, there are some patterns, such as oscillons, ${ }^{10}$ which are not explained so far by the weakly nonlinear analysis. In the effort to understand these patterns, numerical simulation of the Faraday waves plays a complementary role.

For this reason, we develop a method of numerical simulation of the two-frequency forced Faraday waves, which is consistent with the experiments. Specifically, we here simulate three patterns observed in the experiments by Arbell and Fineberg. ${ }^{9,10}$ In particular, the rhomboidal pattern does not appear in the single-frequency forced Faraday waves. In order to validate the simulations, we compare our results with the linear stability analysis of two frequency Faraday waves. ${ }^{18} \mathrm{Next}$, in the nonlinear regime, we reproduce the square pattern and the hexagonal pattern with the same physical parameters as the respective experiments. After that we reproduce and study the rhomboidal state. During the simulations, we compare two kinds of level-set methods: one is the original implementation ${ }^{29,30}$ and the other is the level-set method with Lagrangian particles (particle level-set method). ${ }^{31}$ Finally, we discuss the difficulty of simulating other patterns observed in the experiments. 
The organization of the paper is the following. In Sec. II, we describe the fluid dynamical equations of the Faraday waves, the two level-set methods, and numerical discretization of the equations. The numerical results are presented in Sec. III. More specifically, comparisons of the simulation with the linear analysis and simple patterns such as square and hexagonal patterns are presented in Secs. III A and III B. The simulation of the rhomboidal states is shown in Sec. III C. In Sec. III D, we compare the original level-set method and the particle level-set method. Our summary and discussion are in Sec. IV.

\section{EQUATIONS AND NUMERICAL METHOD}

In this section, we describe our numerical method for the governing equations and the boundary conditions used for simulating Faraday waves oscillated by the two-frequency forcing.

\section{A. Navier-Stokes equations}

Faraday waves occur on the interface between an upper and a lower immiscible fluids. We employ the one-fluid description of the problem. Numerically, we simulate the dynamics in both fluid layers. The incompressible Navier-Stokes equations are written as

$$
\begin{aligned}
\nabla \cdot \boldsymbol{u} & =0, \\
\rho D_{t} \boldsymbol{u} & =-\nabla p+\rho \boldsymbol{G}+\nabla \cdot \eta\left(\nabla \boldsymbol{u}+\nabla \boldsymbol{u}^{T}\right)+\boldsymbol{s} .
\end{aligned}
$$

Here, $D_{t}, p$, and $\boldsymbol{u}$ are the material derivative, the pressure, and the velocity, respectively, and $s, \rho$, and $\eta$ are the surface force, the density, and the viscosity, respectively. The vector $\boldsymbol{G}$ is the gravitational term in the reference frame of the container,

$$
\boldsymbol{G}=\left(-g+A_{1} \cos \left(m \omega_{0} t\right)+A_{2} \cos \left(n \omega_{0} t+\theta\right)\right) \boldsymbol{e}_{z},
$$

where $g, A_{1}, A_{2}, \omega_{0}, \theta$, and $\boldsymbol{e}_{z}$ are the gravitational acceleration, the amplitude of the first periodic forcing, the amplitude of the second periodic forcing, the base angular frequency of the periodic forcing, the phase shift between the two modes, and the unit vector in the vertical $z$-direction, respectively. In this paper, we set the integers $m$ and $n$ to 2 and 3. We also use the notations $\omega_{1}=m \omega_{0}=2 \omega_{0}, \omega_{2}=$ $n \omega_{0}=3 \omega_{0}$.

On the top and bottom boundaries, no-slip boundary conditions are assumed. For the horizontal direction, we assume periodic boundary conditions. The interface location $z=\zeta(x, y, t)$ obeys the kinematic boundary condition. In term of this $\zeta$, the density $\rho$ and $\eta$ are written as

$$
(\rho, \eta)=\left\{\begin{array}{l}
\left(\rho_{t}, \eta_{t}\right), z>\zeta(x, y, t), \\
\left(\rho_{b}, \eta_{b}\right), z \leq \zeta(x, y, t),
\end{array}\right.
$$

where $\rho_{t}, \eta_{t}$ are the density and the viscosity of the top fluid and $\rho_{b}, \eta_{b}$ are the density and the viscosity of the bottom fluid.

In this sharp interface description, the density and the viscosity change discontinuously at the dynamically evolving interface. This situation is a challenge for numerical simulations. To circumvent this difficulty, various numerical methods have been proposed, such as the volume-of-fluid methods, the level-set methods, and the front-tracking methods, just to name a few. ${ }^{15,32}$ In this study, we adopt the level-set method. The reason is as follows. The level-set method has a high numerical accuracy of the normal vector and the curvature of the interface, hence adequate for the gravity-capillary waves. However, it is well known that the level-set method does not have good mass conservation properties. ${ }^{32}$ A number of improvements have been proposed. ${ }^{30-33}$ Among them, we use the level-set method corrected with Lagrangian particles, the so-called particle level-set method, to ensure volume conservation. ${ }^{31}$ This conservation problem is discussed in detail in Sec. III D.

In the following Sec. II B, we describe the level-set method without the particles; here, we call the original level-set method and the particle level-set method and their numerical discretizations. The description of the discretization of the Navier-Stokes equations follows later. 


\section{B. Level-set method}

\section{Level-set function}

We use the level-set approach ${ }^{29}$ to describe the interface motion. Here, the level-set function $\phi(x, t)$, the signed distance from the interface, indicates the interface. We define $\phi>0$ in the top fluid and $\phi<0$ in the bottom fluid. The level-set function obeys the following equation:

$$
\partial_{t} \phi+(\boldsymbol{u} \cdot \nabla) \phi=0,
$$

which is discretized with the 5th-order weighted essentially non-oscillatory (WENO) scheme ${ }^{34}$ and integrated in time through the 3rd-order total variation diminishing (TVD) Runge-Kutta method. ${ }^{34}$

\section{Reinitialization of level-set function}

It is known that the analytic integration of Eq. (5) does not ensure that $\phi(x, t)$ is the signed distance function from the interface. By definition, being the signed distance function requires $|\nabla \phi|=1$. However, this unit gradient condition is not satisfied since the Lagrange derivative of $|\nabla \phi|^{2}$ is not zero but $D_{t}|\nabla \phi|^{2}=-2(\nabla \phi)(\nabla \boldsymbol{u})(\nabla \phi)$. To enforce the condition (in practice, we do so just around the interface), the distance function is re-initialized at each time step from the following initial value problem with the virtual time $\tau:^{30}$

$$
\begin{aligned}
& \frac{\partial d}{\partial \tau}=\operatorname{sgn}(\phi)(1-|\nabla d|)+\lambda f(\phi), \\
& d(x, y, z, \tau=0)=\phi(x, y, z) .
\end{aligned}
$$

Although we call $\tau$ virtual time, its dimension is length. Ideally, the function $d(\boldsymbol{x}, \tau)$ as $\tau \rightarrow \infty$ gives the corrected signed distance function for all the computational domain. Here, we set $\phi(x, t)=d(\boldsymbol{x}, \tau=$ $\tau_{l}$ ) for some value $\tau_{l}$. This $\tau_{l}$ corresponds to the largest distance from the interface to which we demand $\phi$ be the signed distance. In this paper, we use $\tau_{l}=\epsilon$, where $\epsilon$ is the half-width of the diffuse interface and set to $2 \Delta z$, where $\Delta z$ is the grid spacing in the vertical $z$-direction. The functions $\lambda(\boldsymbol{x}), f(\phi)$ in Eq. (6) are given as

$$
\begin{aligned}
\lambda(\boldsymbol{x}) & =-\frac{\int_{\Omega(x)} H^{\prime}(\phi) L(\phi, d) d \boldsymbol{x}}{\int_{\Omega(x)} H^{\prime}(\phi) f(\phi) d \boldsymbol{x}}, \\
f(\phi) & =H^{\prime}(\phi)|\nabla \phi|, \\
H^{\prime}(\phi) & =\frac{d H}{d \phi}, \\
H(\phi) & = \begin{cases}0, & \text { if } \phi<-\epsilon, \\
\frac{1}{2}\left\{1+\frac{\phi}{\epsilon}+\frac{1}{\pi} \sin \left(\frac{\pi \phi}{\epsilon}\right)\right\}, & \text { if }-\epsilon \leq \phi \leq \epsilon, \\
1, & \text { if } \phi>\epsilon,\end{cases}
\end{aligned}
$$

where $\Omega(x)$ is a small region centered at the point $x, L(\phi, d)=\operatorname{sgn}(\phi)(1-|\nabla d|), \epsilon=2 \Delta z$ is the prescribed interface width, $H(\phi)$ is the smoothed Heaviside function, and $H^{\prime}(\phi)$ is the smoothed delta function.

Numerically, the reinitialization is done in the following way. First, we ignore the term $\lambda(\boldsymbol{x}) f(\phi)$ in the Eq. (6) and solve

$$
\frac{\partial d}{\partial \tau}=\operatorname{sgn}(\phi)(1-|\nabla d|)
$$

where the discretization in space is the same as that of Eq. (5). The integration in the virtual time is discretized as follows 


$$
\begin{aligned}
d^{\prime} & =d^{n}+\Delta \tau L\left(\phi, d^{n}\right), \\
d^{*} & =\frac{1}{4}\left(3 d^{n}+d^{\prime}+\Delta \tau L\left(\phi, d^{\prime}\right)\right), \\
d^{\prime n+1} & =\frac{1}{3}\left(d^{n}+2\left(d^{*}+\Delta \tau L\left(\phi, d^{*}\right)\right)\right),
\end{aligned}
$$

where $\Delta \tau=0.5 \min (\Delta x, \Delta y, \Delta z)$.

Second, we calculate $\lambda(\boldsymbol{x})$ according to the following equation:

$$
\lambda_{i j k}=\frac{-\int_{\Omega_{i j k}} H^{\prime}(\phi) \frac{d^{\prime n+1}-\phi}{\Delta \tau} d \boldsymbol{x}}{\int_{\Omega_{i j k}} H^{\prime}(\phi) f(\phi) d \boldsymbol{x}} .
$$

Here, $\lambda_{i j k}$ denotes $\lambda\left(x_{i j k}\right)$ on the grid point $\boldsymbol{x}_{i j k}$ specified by the index $(i, j, k)$. The integral range $\Omega_{i j k}$ describes the cell region associated with the grid point. For the three dimensional case, by following the two-dimensional version, ${ }^{35}$ we discretize the integral of some function $g(x)$ in the cell as

$$
\begin{aligned}
\int_{\Omega_{i j k}} g(\boldsymbol{x}) d \boldsymbol{x}= & \frac{\Delta x \Delta y \Delta z}{1512}\left[9 \left(g_{i+1, j+1, k}+g_{i+1, j-1, k}\right.\right. \\
& +g_{i-1, j+1, k}+g_{i-1, j-1, k}+g_{i, j+1, k+1} \\
& +g_{i, j+1, k-1}+g_{i, j-1, k+1}+g_{i, j-1, k-1} \\
& +g_{i+1, j, k+1}+g_{i+1, j, k-1}+g_{i-1, j, k+1} \\
& \left.+g_{i-1, j, k-1}\right)+88\left(g_{i+1, j, k}+g_{i-1, j, k}\right. \\
& +g_{i, j+1, k}+g_{i, j-1, k}+g_{i, j, k+1} \\
& \left.\left.+g_{i, j, k-1}\right)+876 g_{i, j, k}\right],
\end{aligned}
$$

where $\Delta x, \Delta y, \Delta z$ are the grid spacings along the $x, y, z$ directions.

Finally, $d^{n+1}$ is calculated by

$$
d^{n+1}=d^{\prime n+1}+\Delta \tau \lambda_{i j k} H^{\prime}(\phi)|\nabla \phi| .
$$

In practice, we take the total number of the virtual time steps as $\tau_{l} / \Delta \tau \simeq 4$.

\section{Particle level-set method}

In order to improve the volume conservation of the level-set method, it has been proposed to utilize Lagrangian information to correct the level-set function by adding marker particles near the interface. Our procedure of the particle level-set method is basically the same as that of Enright et al. ${ }^{31}$ The differences are in the error correction and the reseeding strategy.

\section{Initialization of particles}

The marker particles are spread in the neighborhood of the interface, in which $|\phi|<3 \max (\Delta x$, $\Delta y, \Delta z)$ is satisfied. The number of particles in each cell is set to 64. A marker particle has sign $s_{p}=1$ or -1 and the radius $r_{p}$. There are a number of strategies for setting the sign and radius. One simple strategy is to set the sign to that of the level-set function at the particle and the radius to the absolute value of the level-set function. However, we follow the more sophisticated strategy proposed by Enright $e t$ al. to improve numerical results.

The strategy is as follows. Initially, the particle's sign is set randomly. In order to have the same sign between the particle and the level-set function, the particles at $\boldsymbol{x}_{p},(p=1,2,3, \ldots)$ are iteratively moved by the following recurrence relation:

$$
x_{p}^{n+1}=x_{p}^{n}+2^{-n}\left(\phi_{\text {goal }}-\phi\left(x^{n}\right)\right) \boldsymbol{N}\left(x_{p}^{n}\right),
$$

where $N\left(x_{p}^{n}\right)=\frac{\nabla \phi\left(x_{p}^{n}\right)}{\left|\nabla \phi\left(x_{p}^{n}\right)\right|}$ is the normal vector. Here, $\phi_{\text {goal }}$ is set as follows. The $\operatorname{sign}, \operatorname{sgn}\left(\phi_{p}\right)$, is set to have the same as that of the particle $s_{p}$. In addition, the absolute value is chosen to be a 
uniformly distributed random variable in the range $b_{\min }<\left|\phi_{\text {goal }}\right|<b_{\max }$. In this study, $b_{\min }$ is set to $0.1 \min (\Delta x, \Delta y, \Delta z)$ and $b_{\max }$ is set to $3 \max (\Delta x, \Delta y, \Delta z)$. Each particle is moved repeatedly by Eq. (18) until it satisfies the condition $b_{\min }<s_{p} \phi\left(\boldsymbol{x}_{p}\right)<b_{\max }$. Finally, each particle radius is set according to

$$
r_{p}= \begin{cases}r_{u} & s_{p} \phi\left(\boldsymbol{x}_{p}\right)>r_{u}, \\ s_{p} \phi\left(\boldsymbol{x}_{p}\right) & r_{l}<s_{p} \phi\left(\boldsymbol{x}_{p}\right)<r_{u}, \\ r_{l} & s_{p} \phi\left(\boldsymbol{x}_{p}\right)<r_{l},\end{cases}
$$

where $r_{l}$ and $r_{u}$ are lower and upper limits of particle radius to prevent the creation of particles which are too small or too large. We use $r_{l}=0.1 \min (\Delta x, \Delta y, \Delta z)$ and $r_{u}=5 r_{l}$. The particle radius $r_{p}$ is used to correct the level-set function later. After this procedure, the positive particles at position $\boldsymbol{x}_{p}$ are in the $\phi\left(\boldsymbol{x}_{p}\right)>0$ side (the top fluid) and the negative particles are in the $\phi\left(\boldsymbol{x}_{p}\right)<0$ side (the bottom fluid). The envelope formed by the circles of the same-sign particles coincides with the interface $\phi=0$.

\section{Advection of particles}

Each particle at position $\boldsymbol{x}_{p}(t)$ is advected by

$$
\frac{d \boldsymbol{x}_{p}(t)}{d t}=\boldsymbol{u}\left(\boldsymbol{x}_{p}(t), t\right)
$$

The velocity at the particle position $\boldsymbol{u}\left(\boldsymbol{x}_{p}(t), t\right)$ is calculated with trilinear interpolation from the velocity vectors on the nearby cell faces. The 3rd order TVD Runge-Kutta method is used to integrate Eq. (20) in time.

\section{Error correction of level-set function}

As a result of the advection Eq. (20), some particles move across the interface $\phi=0$. Such escaped particles are used to correct the level-set function $\phi$ in the following manner. First, particles placed on the wrong side $\left(\phi\left(\boldsymbol{x}_{\boldsymbol{p}}\right) \times s_{p}<0\right)$ are considered to have escaped. Second, we introduce the signed distance function between the escaped particle and a point $\boldsymbol{x}$, which is calculated with the particle radius $r_{p}$ as

$$
\phi_{p}(\boldsymbol{x})=s_{p}\left(r_{p}-\left|\boldsymbol{x}-\boldsymbol{x}_{p}\right|\right) .
$$

This signed distance is positive $\left(\phi_{p}(\boldsymbol{x})>0\right)$ if the point $\boldsymbol{x}$ is within the positive ball $\left(s_{p}=1\right)$ of radius $r_{p}$ centered on $\boldsymbol{x}_{p}$. The distance function corrected by the escaped positive (negative) particle $\phi_{+}(\boldsymbol{x})\left(\phi_{-}(\boldsymbol{x})\right)$ is calculated from

$$
\begin{aligned}
& \phi_{+}(\boldsymbol{x})=\max _{\forall p \in E^{+}}\left(\phi_{p}, \phi(\boldsymbol{x})\right), \\
& \phi_{-}(\boldsymbol{x})=\min _{\forall p \in E^{-}}\left(\phi_{p}, \phi(\boldsymbol{x})\right) .
\end{aligned}
$$

Here, $E^{+}$and $E^{-}$denote the sets of the escaped positive and negative particles. Finally, the level-set function $\phi(\boldsymbol{x})$ is corrected as

$$
\phi(\boldsymbol{x})= \begin{cases}\phi_{+}(\boldsymbol{x}) & \text { if }\left|\phi_{+}(\boldsymbol{x})\right| \leq\left|\phi_{-}(\boldsymbol{x})\right|, \\ \phi_{-}(\boldsymbol{x}) & \text { if }\left|\phi_{-}(\boldsymbol{x})\right|<\left|\phi_{+}(\boldsymbol{x})\right| .\end{cases}
$$

Ideally, after this correction of the distance function, all the particles tagged as escaped have the same sign as the corrected distance function. However, with our implementation of the correction in the preliminary calculations, we find that some particles do not have the same sign of the corrected distance function. If we use such particles with the wrong sign in the next correction process, the interface becomes nearly singular, which we consider a numerical artifact. Therefore, we ignore such escaped particles in the later correction processes. The point differs from the usual procedure of the particle level-set method. ${ }^{31}$ 


\section{Reseeding of particles}

Generally, as a result of advection of the particles by a flow, some regions lack sufficient particles to correct the level-set function. We reseed the particles where needed. Specifically, in the cells near the interface $\left(|\phi(\boldsymbol{x})| \leq b_{\max }\right)$, we keep the number of particles in a cell to 64 by adding particles for cells which particles exit or deleting particles for cells which particles enter. In our simulation, the reseeding procedure is executed with the following two strategies. The first strategy is that the reseeding is done after 40 time steps from the previous reseeding. The second strategy is that the reseeding is done when the surface area of the interface increases by $30 \%$ after the previous reseeding time. The surface area is calculated from

$$
\begin{aligned}
A & =\int \delta(\phi)|\nabla \phi| d x, \\
\delta(\phi) & = \begin{cases}0, & 1 \\
\frac{1}{2 \epsilon}\left(1+\cos \left(\frac{\pi \phi}{\epsilon}\right)\right), & |\phi| \leq \epsilon,\end{cases}
\end{aligned}
$$

where the smoothed delta function $\delta(\phi)$ is the same as $H^{\prime}(\phi)$ of Eq. (9).

In summary, the one-step update of the level-set function with particles is carried out by the following steps. ${ }^{31}$

1. The level-set function is advected by Eq. (5).

2. The particles are advected by Eq. (20).

3. The error of the level-set function is corrected by the procedure described in the Sec. II C 3.

4. The level-set function is reinitialized as described in Sec. II B 2.

5. The error of the level-set function is once more corrected by the particles as described in Sec. II C 3.

For the original level-set method without particles, the second, third, and fifth processes are omitted.

\section{Discretization of Navier-Stokes equations}

We use the following temporal discretization of incompressible Navier-Stokes Eqs. (1) and (2) with the projection method and with adaptive time stepping:

$$
\begin{aligned}
\boldsymbol{u}^{*}= & \boldsymbol{u}^{n}+\Delta t^{n}[ \\
& -\left(1+\frac{\Delta t^{n-1}}{\Delta t^{n}}\right) \boldsymbol{A}^{n}+\frac{\Delta t^{n-1}}{\Delta t^{n}} \boldsymbol{A}^{n-1} \\
& +\left(1+\frac{\Delta t^{n-1}}{\Delta t^{n}}\right) \boldsymbol{D}_{e}^{n}-\frac{\Delta t^{n-1}}{\Delta t^{n}} \boldsymbol{D}_{e}^{n-1} \\
& \left.+\frac{1}{2}\left(\boldsymbol{D}_{i}^{*}+\boldsymbol{D}_{i}^{n}\right)+\boldsymbol{G}^{n}+\boldsymbol{S}^{n+1}\right], \\
\boldsymbol{u}^{n+1}= & \boldsymbol{u}^{*}+\Delta t^{n} \frac{1}{\rho^{n}} \nabla p^{n+1} .
\end{aligned}
$$

Here, the superscript $n$ denotes the value at the $n$-th time step $t^{n}=\sum_{i=1}^{n} \Delta t^{i}$ in which $\Delta t^{i}$ is the time step size for the $i$-th step. We discuss later how to determine them. $\boldsymbol{A}^{n}$ is the advective term, $\boldsymbol{D}_{i}^{n}$ and $\boldsymbol{D}_{i}^{*}$ are the viscous terms involving the same component as on the left-hand-side and at the intermediate step, $\boldsymbol{D}_{e}^{n}$ is the viscous term involving the other components, $\boldsymbol{G}^{n}$ is gravity, and $\boldsymbol{S}^{n}$ is the surface force term. Here, by ${ }^{*}$, we denote the intermediate step. As in the standard way of the projection method, the pressure term $p^{n+1}$ is calculated from the divergence free condition. Equation (28) acted upon by $\nabla \cdot$ becomes

$$
-\frac{\nabla \boldsymbol{u}^{*}}{\Delta t^{n}}=\nabla\left(\frac{1}{\rho^{n}} \nabla p^{n+1}\right)
$$


The advection term $\boldsymbol{A}^{n}$ is described by

$$
\boldsymbol{A}^{n}=\boldsymbol{u}^{n} \cdot \nabla \boldsymbol{u}^{n} .
$$

The $x$-component of the viscous term $\boldsymbol{D}_{i}^{n}$ to be treated implicitly is

$$
D_{i x}^{n}=\frac{1}{\rho^{n}}\left[\nabla\left(\eta^{n} \nabla u_{x}^{n}\right)+\frac{\partial}{\partial x}\left(\eta^{n} \frac{\partial u_{x}^{n}}{\partial x}\right)\right] .
$$

The viscous term $D_{i x}^{*}$ is defined similarly but with the intermediate velocity $\boldsymbol{u}^{*}$. The $x$-component of the viscous term $\boldsymbol{D}_{e}^{n}$ to be treated explicitly is

$$
D_{e x}^{n}=\frac{1}{\rho^{n}}\left[\frac{\partial}{\partial y}\left(\eta^{n} \frac{\partial u_{y}^{n}}{\partial x}\right)+\frac{\partial}{\partial z}\left(\eta^{n} \frac{\partial u_{z}^{n}}{\partial x}\right)\right] .
$$

Other components of the viscous terms are described in the same manner. The gravitational term $\boldsymbol{G}^{n}$ is

$$
\boldsymbol{G}^{n}=\left(-g+A_{1} \cos \left(\omega_{1} t^{n}\right)+A_{2} \cos \left(\omega_{2} t^{n}+\theta\right)\right) \boldsymbol{e}_{z} .
$$

The surface force term $S^{n}$ is

$$
\boldsymbol{S}^{n}=\sigma \kappa^{n} \boldsymbol{n}^{n}, \quad \boldsymbol{n}^{n}=\frac{\nabla \phi^{n}}{\left|\nabla \phi^{n}\right|}, \quad \kappa^{n}=\nabla \cdot \boldsymbol{n}^{n} .
$$

Regarding the spacial discretizations, the advection term, $\boldsymbol{A}^{n}$,is discretized with the 2nd-order essentially non-oscillatory (ENO) scheme. The other derivative terms in $\boldsymbol{D}_{i}^{*}, \boldsymbol{D}_{i}^{n}, \boldsymbol{D}_{e}^{n}, \boldsymbol{n}^{n}, \kappa^{n}$ are discretized with the 2nd-order central difference scheme.

Concerning the boundary conditions, we assume periodic boundary conditions in the horizontal directions ( $x$ and $y$ directions). For the vertical $z$ direction, we assume the non-slip condition at $z=0, L_{z}$,

$$
\left.\boldsymbol{u}\right|_{z=0, L_{z}}=\mathbf{0} .
$$

The boundary condition for the pressure in solving Poisson equation (29) is

$$
\left.\frac{\partial p}{\partial z}\right|_{z=0, L_{z}}=0 .
$$

For calculating $\boldsymbol{u}^{*}$ in Eq. (27), the localized incomplete LU (ILU) factorization preconditioned biconjugate gradient stabilized (BiCGSTAB) method ${ }^{36}$ is adopted and the Poisson equation of the pressure is solved by the multigrid preconditioned BiCGSTAB method. ${ }^{36}$

Finally, we describe how we determine the variable time step size $\Delta t^{i}$ which is determined by

$$
\Delta t^{i}=c \min _{\boldsymbol{x}}\left(\Delta t_{S}, \Delta t_{f}^{i}(\boldsymbol{x}), \Delta t_{\eta}(\boldsymbol{x}), \Delta t_{c f l}^{i}(\boldsymbol{x})\right),
$$

where we set the safety constant $c=0.40$. Here, $\Delta t_{S}, \Delta t_{f}$, and $\Delta t_{\eta}$ are the time scales of surface force, the vertical vibration, and the viscosity, respectively. The time scale $\Delta t_{c f l}$ concerns the CourantFriedrichs-Lewy (CFL) condition. These reference time scales are defined as

$$
\begin{aligned}
& \Delta t_{S}=\sqrt{\frac{\left(\rho_{t}+\rho_{b}\right) d h^{3}}{4 \pi \sigma}}, \quad(d h=\min (\Delta x, \Delta y, \Delta z)), \\
& \Delta t_{f}^{i}(\boldsymbol{x})=\sqrt{\frac{d h}{\boldsymbol{G}^{i} \cdot \boldsymbol{e}_{z}}}, \quad \Delta t_{\eta}(\boldsymbol{x})=\frac{\rho}{\eta} \frac{1}{\frac{1}{\Delta x^{2}}+\frac{1}{\Delta y^{2}}+\frac{1}{\Delta z^{2}}}, \\
& \Delta t_{c f l}^{i}(\boldsymbol{x})=\frac{1}{\frac{u_{x}^{i}}{\Delta x}+\frac{u_{y}^{i}}{\Delta y}+\frac{u_{z}^{i}}{\Delta z}} .
\end{aligned}
$$

Typically, $\Delta t_{S}$ is the smallest in our all simulations. 


\section{NUMERICAL RESULTS}

Our goal in this paper is numerical simulation of the $2 k$ rhomboidal states observed in the laboratory experiments by Arbell and Fineberg ${ }^{9,10}$ To the best of our knowledge, the rhomboidal states have not previously been obtained in numerical simulations of the Navier-Stokes equations. In particular, we use the same bulk fluid parameters as the experiments. The only difference is the geometry of the system, i.e., the domain size and the boundary conditions. In the simulations, we apply periodic boundary conditions in the horizontal directions, with which we can reduce numerical cost by not simulating many repeated patterns in the computational domain. While the experiments are conducted in an open container, we assume the presence of a rigid wall above the top fluid, on which the no-slip boundary condition is applied. This setting is numerically easier than simulating the top-fluid motion in a semi-infinite domain. We assume that the top-fluid height is four times larger than the bottom-fluid depth.

Before presenting the simulation, we first describe the validation of our fully nonlinear simulation with the above mentioned geometry by comparing with the linear stability analysis of two-frequency forced Faraday waves. ${ }^{18}$ This validation process is the same as Périnet et al. ${ }^{16}$ The second test then is to reproduce the square and hexagonal patterns observed in the same two-frequency forced experiments. ${ }^{9,10}$ A direct numerical simulation of the square and hexagon patterns for the single-frequency forced Faraday waves is performed by Périnet et al. ${ }^{16,37}$ The result on the rhomboidal states is presented after the validations.

\section{A. Comparison with linear stability analysis}

We now compare the critical amplitudes of the oscillations calculated with the fully nonlinear numerical simulation with those calculated with the linear stability analysis. We write the twofrequency forcing as $a\left(\cos (\chi) \cos \left(\omega_{1} t\right)+\sin (\chi) \cos \left(\omega_{2} t+\theta\right)\right)$. In the linear analysis, once we fix the physical parameters as shown in Table I and the mixing angle $\chi$, the critical value of $a$, denoted as $a_{c}$, and the associated critical wave number can be calculated. ${ }^{18,19}$ Here, we assume that either harmonic frequency $\left(\omega_{1}, \omega_{2}\right)$ or sub-harmonic frequency $\left(\omega_{1} / 2, \omega_{2} / 2\right)$ gives the lowest critical value. The critical amplitudes $a_{1 c}=a_{c} \cos (\chi)$ and $a_{2 c}=a_{c} \sin (\chi)$ for the mixing angle $\chi$ from $0^{\circ}$ to $90^{\circ}$ are shown as the solid line in Fig. 1.

Meanwhile, with the particle level-set simulation, we determine the critical amplitudes by adding small perturbations to basic modes for ten different values of the mixing angle, $\chi=$ $0^{\circ}, 10^{\circ}, 20^{\circ}, \ldots, 90^{\circ}$. The results are denoted as points in Fig. 1 . The way to estimate the critical amplitudes $a_{1 c}$ and $a_{2 c}$ in the nonlinear simulation is as follows: (i) the perturbation is added to the normal mode whose wavenumber is set to the critical wave number $\left(k_{c}\right)$ calculated from the linear analysis. More precisely, the perturbation, $\xi \sin \left(k_{c} x\right)$, is added to the flat interface, where the perturbation amplitude $\xi$ is set to $2 \times 10^{-2} L_{z}$. (ii) the interface height $\zeta(x, y, t)$ at the center of the

TABLE I. Parameter values for the linear stability analysis. These parameters (except for $L_{z}$ and $\theta$ ) are identical to the experiment by Arbell and Fineberg. ${ }^{10}$

\begin{tabular}{lcc}
\hline \hline$\rho_{t}$ & 1.293 & $\left(\mathrm{kgm}^{-3}\right)$ \\
$\rho_{b}$ & $9.500 \times 10^{2}$ & $\left(\mathrm{kgm}^{-3}\right)$ \\
$\eta_{t}$ & $1.822 \times 10^{-5}$ & $\left(\mathrm{~kg} \mathrm{~m}^{-1} \mathrm{~s}^{-1}\right)$ \\
$\eta_{b}$ & $2.185 \times 10^{-2}$ & $\left(\mathrm{kgm}^{-1} \mathrm{~s}^{-1}\right)$ \\
$\omega_{1}=2 \omega_{0}$ & $3.770 \times 10^{2}$ & $\left(\mathrm{~s}^{-1}\right)$ \\
$\omega_{2}=3 \omega_{0}$ & $5.655 \times 10^{2}$ & $\left(\mathrm{~s}^{-1}\right)$ \\
$\theta$ & 0 & $(\mathrm{rad})$ \\
$\sigma$ & $2.150 \times 10^{-2}$ & $\left(\mathrm{kgm}^{-1}\right)$ \\
$g$ & 9.807 & $\left(\mathrm{~ms}^{-2}\right)$ \\
$L_{z}$ & $1.00 \times 10^{-2}$ & $(\mathrm{~m})$ \\
Bottom-fluid depth & $2.00 \times 10^{-3}$ & $(\mathrm{~m})$ \\
\hline \hline
\end{tabular}




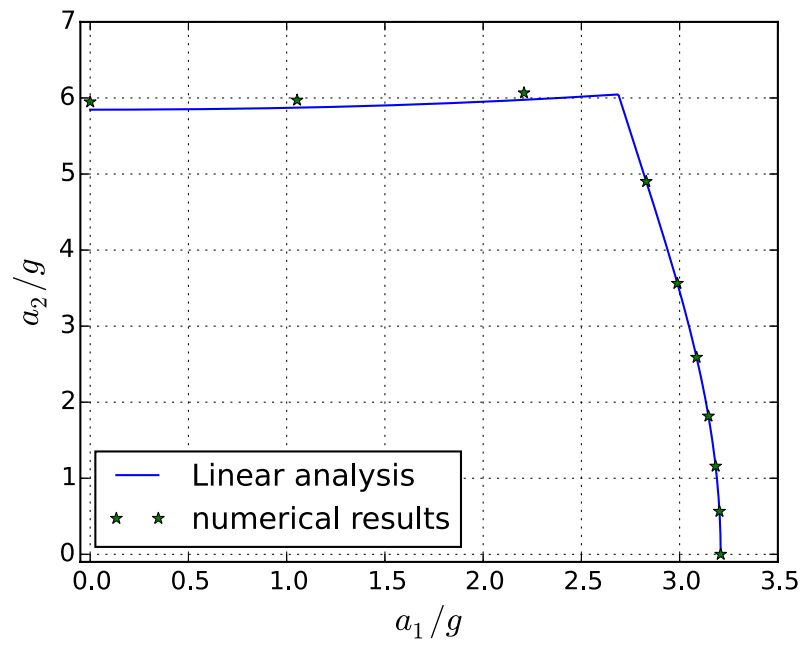

FIG. 1. Comparison of the critical vibration amplitudes obtained with the numerical simulation to those calculated with the linear stability analysis.

calculation domain is monitored throughout the simulation for given $a$ and $\chi$. The interface height $z=\zeta(x, y, t)$ is calculated from the zero points of the level-set function $\phi(x, y, z, t)=0$. We perform such simulations by changing $a$ and estimate the critical value $a_{c}$. More precisely, we take the absolute relative difference between the two peak interface heights at $t=4.44 T_{v}$ and $6.44 T_{v}$, where $T_{v}=2 \pi / \omega_{0}=2 \pi /\left(\omega_{1} / 2\right)=2 \pi /\left(\omega_{2} / 3\right)$. Then, $2 T_{v}$ is the minimal period of the two waves with the subharmonic frequencies $\omega_{1} / 2$ and $\omega_{2} / 2$. If the difference is smaller than $10^{-3}$, this $a$ is regarded as the critical amplitude of the level-set numerical simulation.

As shown in Fig. 1, the critical amplitudes calculated with the level-set simulation tend to be greater than those calculated with the linear analysis in the $a_{2}$ dominant region, namely, $a_{1} / g<2.6$. The absolute relative error between the linear analysis (line) and the simulation (point) in the region is about 0.02 . The agreement between the two results is hence obtained with two-digit accuracy.

\section{B. Square and hexagonal patterns}

Having validated the simulation of the two-frequency forced Faraday waves in the linear regime, we now move to two nonlinear cases: the square and hexagonal patterns. Note that the two patterns are also observed in the single-frequency Faraday waves.

First, we reproduce the square pattern observed in the experiments by Arbell and Fineberg. ${ }^{9,10}$ The physical parameters are shown in Table II. We select the amplitudes of the forcing $A_{1}$ and $A_{2}$ according to the following reasons: (i) we aim to conduct the simulation in the weakly nonlinear regime; (ii) we aim to set the values to be neither close to nor far from the bicritical point. The selected values of $A_{1}$ and $A_{2}$ in Table II are of course in the square-pattern domain of the phase diagram obtained experimentally. ${ }^{10}$ However, the geometry of the simulation is different. We set the lateral dimensions of the computational domain so that it includes one square $2 \pi / k_{1}=\lambda_{1}$, where $k_{1}$ is the critical wave

TABLE II. Parameter values for the square pattern. The number of the grid points for each direction is $N_{x}, N_{y}, N_{z}$. The other parameters are the same as the Table I.

\begin{tabular}{lcc}
\hline \hline$L_{x}$ & $4.373 \times 10^{-3}$ & $(\mathrm{~m})$ \\
$L_{y}$ & $4.373 \times 10^{-3}$ & $(\mathrm{~m})$ \\
$A_{1}$ & $2.000 \times 10^{1}$ & $\left(\mathrm{~ms}^{-2}\right)$ \\
$A_{2}$ & $6.000 \times 10^{1}$ & $\left(\mathrm{~ms}^{-2}\right)$ \\
$N_{x} \times N_{y} \times N_{z}$ & $64 \times 64 \times 64$ & \\
\hline \hline
\end{tabular}


number, which is found to be $1.436 \times 10^{3}\left(\mathrm{~m}^{-1}\right)$ from the linear stability analysis described in Sec. III A. In other words, we set the computational domain to a square box with $L_{x}=L_{y}=\lambda_{1}$. This setting is the minimal computational domain which supports the periodic square pattern. The number of grid points used in each horizontal direction is denoted by $N_{x}=N_{y}$. The square pattern consists of the four discrete Fourier modes shown as black dots in Fig. 6(a). These modes, called resonant modes, are on the circle of radius $k_{1}$. The amplitude of the resonant wavevectors can be calculated from the linear stability analysis. The direction of those can be estimated from the experimental data. The experimental information of the direction is trivial in the case of the square pattern. However, the information becomes crucial in the case of more complex patterns as we will see later. The resultant grid on the Fourier space is shown in Fig. 6(a).

We start the simulation with zero velocity everywhere and the perturbed flat interface. The perturbation of the interface is given in terms of the Fourier modes $\hat{\zeta}(\boldsymbol{k}, t=0)$ for the wavenumber domain defined by $0<\left|k_{x}\right|<0.5 \max \left(k_{x}\right)=\pi N_{x} /\left(2 L_{x}\right)$ and $0<\left|k_{y}\right|<0.5 \max \left(k_{y}\right)=\pi N_{y} /\left(2 L_{y}\right)$. The real and imaginary parts of $\hat{\zeta}(\boldsymbol{k})$ in the range are set by independently and identically distributed random variables with a uniform distribution between $-1 / 2$ and $1 / 2$. The zero Fourier mode is set $\hat{\zeta}(\boldsymbol{k}=\mathbf{0})=$ $0.2 L_{z}$. We finally transform $\hat{\zeta}(\boldsymbol{k})$ in the physical space and multiply the perturbation by an arbitrary factor so that $\max \left(\left|\zeta(x)-0.2 L_{z}\right|\right)=5.0 \times 10^{-2} L_{z}$.

We use here both the original level-set method and the particle level-set method for comparison.

As shown in Fig. 2, indeed, a square pattern is obtained in the simulations. With both the original level-set method and the particle level-set method, we start to recognize the square pattern around $t \sim T_{v}$. In spite of the same appearance of the pattern, the long-time behaviors of the two level-set methods are different. With the particle level-set method, the temporal variation of the interface elevation at a point $(x, y)=\left(0.5 L_{x}, 0.78 L_{y}\right)$ reaches a steady state around $t \sim 38 T_{v}$ as seen in Fig. 3 (solid line). In contrast, with the original level-set method, it does not reach a steady state but keeps increasing as depicted with the dotted line in Fig. 3. However, the square pattern is not destroyed by the unsteadiness up to $t=45 T_{v}$, at which we end the simulation.

To characterize the difference between the original and particle level-set methods, we here introduce two time scales: first pattern recognition time and saturation time. The former is the time we first recognize the expected pattern, which is $T_{v}$ for the square pattern case. The latter is the time needed to reach the steady state, which is $38 T_{v}$ for the particle level-set method. Although these time scales are determined subjectively and are dependent on the initial condition, they play a useful role in comparison between the two level-set methods as we will discuss later.

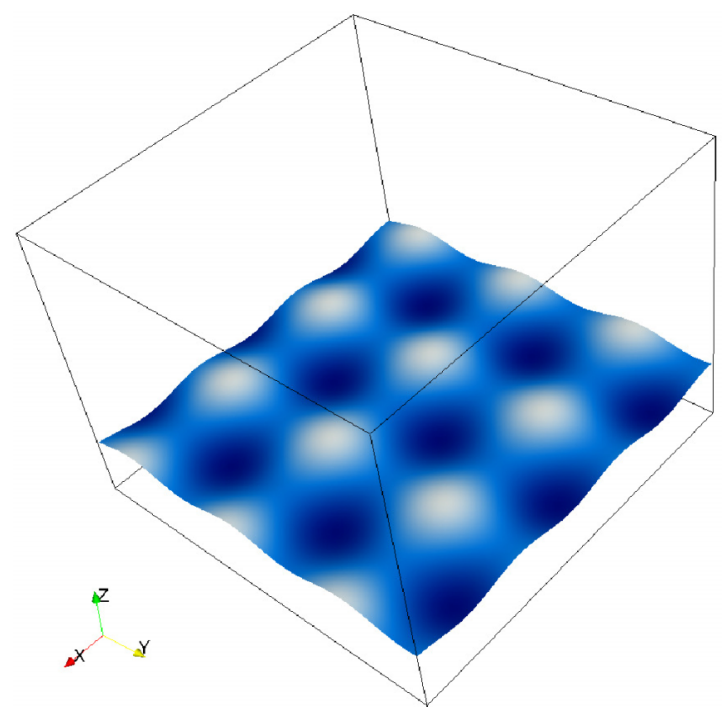

FIG. 2. The interface profile for the square-pattern regime at $t=44.5 T_{v}$. The interface is colored according to its height: the lighter gray (white online) corresponds to higher region and the darker gray (blue online) corresponds to lower region. Each horizontal length of the domain displayed here is three times that of the calculation domain. The aspect ratio of this figure is $\left(3 L_{y}\right) /\left(3 L_{x}\right)=1.000$. Here, we show the result with the particle level-set method. 


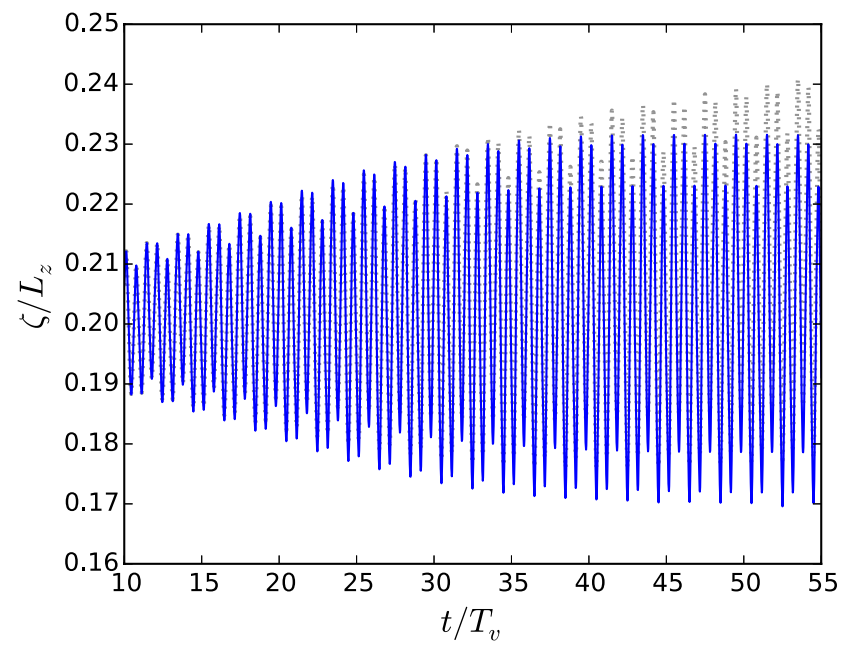

FIG. 3. Temporal variation of the interface height at position $(x, y)=\left(0.5 L_{x}, 0.78 L_{y}\right)$ for the square pattern calculated with the particle level-set method (solid line) and with the original level-set method (dotted line).

Our next target is the hexagonal pattern observed in the experiments. ${ }^{9,10}$ The physical parameters of the simulation are listed in Table III. As in the square pattern case, we set the size of the horizontal domain to the minimal size containing one hexagon. Specifically, with the resonant wavevector $\boldsymbol{k}_{1}^{\prime}$ shown in Fig. 6(b), the lengths are $L_{x}=2 \pi / k_{1 x}^{\prime}$ and $L_{y}=2 \pi / k_{1 y}^{\prime}$. The aspect ratio is $L_{y} / L_{x}=$ $0.5774 \approx 1 / \sqrt{3}$. The numbers of grid points used per wavelength are $\left(\lambda_{1} / L_{x}\right) N_{x}=0.5 N_{x}$ and $\left(\lambda_{1} / L_{y}\right)$ $N_{y}=0.8660 N_{y} \approx \sqrt{3} / 2 N_{y}$. We use the same initial condition as the square pattern case.

The hexagonal pattern is reproduced with both level-set methods. The result with the particle level-set method is shown in Fig. 4. Despite the pattern being the same, the first pattern recognition time is different between the two level-set methods: $6 T_{v}$ for the original level-set method and $19 T_{v}$ for the particle level-set method. The saturation time is $24 T_{v}$ with the particle level-set method as shown in Fig. 5. In contrast, saturation does not occur with the original level-set method during our simulations of length $t=40 T_{v}$. The hexagonal shape of the pattern is maintained in spite of the unsteadiness.

The above results on the square and hexagonal patterns suggest that the original level-set method is not a suitable interface-tracking scheme for Faraday waves. Although the patterns initially emerged with the original level-set method are consistent with the experiment, it is seen that the temporal variation of the interface height does not reach a steady state. This unsteadiness in the long run may change the correctly selected pattern initially into a different shape with the original level-set method. In the simulation of the rhomboidal pattern, the deficiency of the original level-set method appears more seriously as we see in Sec. III C.

\section{Rhomboidal states}

The next pattern we seek to simulate is called the $2 \mathrm{k}$ rhomboidal state observed in the experiment by Arbell and Fineberg. ${ }^{9}$ The pattern is observed around the bicritical point which appears as the sharp tip in Fig. 1. There are two linearly unstable wavenumbers $k_{1}$ and $k_{2}$, hence the name $2 k$ rhomboidal states. As a result of the nonlinear interaction among the resonant modes, a simple resonance

TABLE III. Parameter values for the hexagonal pattern. The other parameters are the same as the Table I.

\begin{tabular}{lcc}
\hline \hline$L_{x}$ & $1.184 \times 10^{-2}$ & $(\mathrm{~m})$ \\
$L_{y}$ & $6.837 \times 10^{-3}$ & $(\mathrm{~m})$ \\
$A_{1}$ & $3.200 \times 10^{1}$ & $\left(\mathrm{~ms}^{-2}\right)$ \\
$A_{2}$ & $3.000 \times 10^{1}$ & $\left(\mathrm{~ms}^{-2}\right)$ \\
$N_{x} \times N_{y} \times N_{z}$ & $112 \times 64 \times 64$ & \\
\hline \hline
\end{tabular}



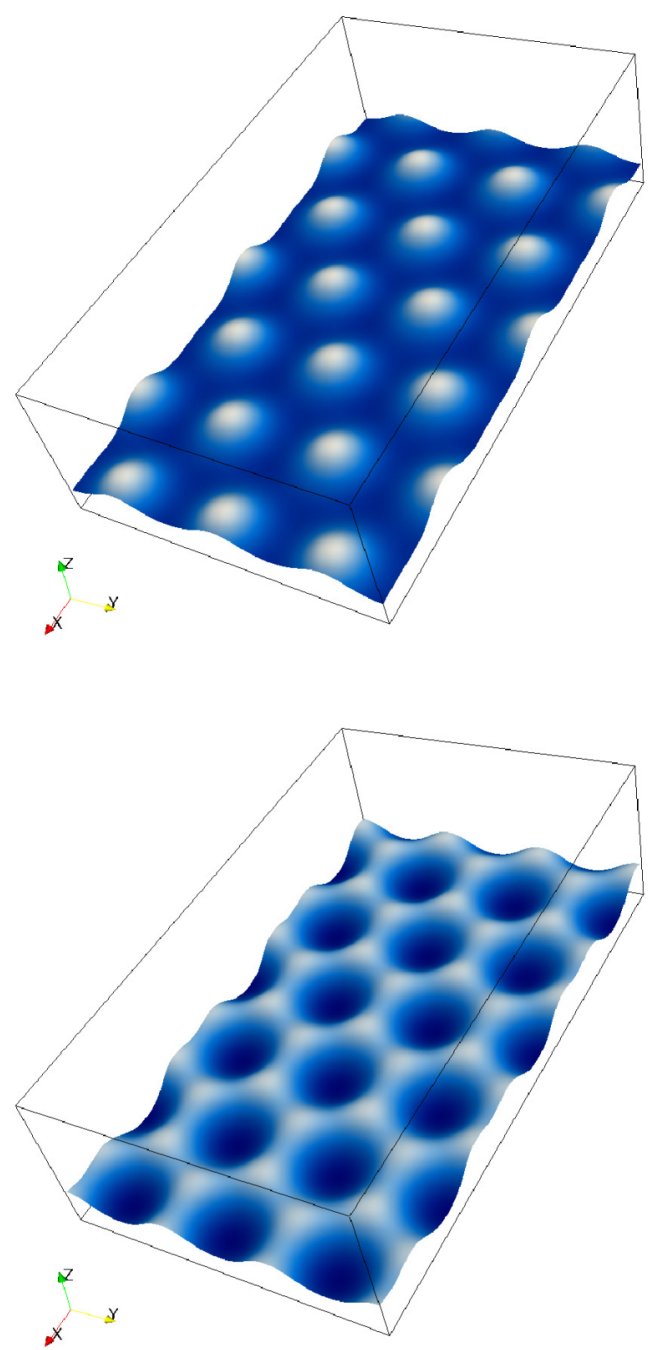

FIG. 4. Interface profile for the hexagonal pattern at $t=27.65 T_{v}$ (above), 28.22 $T_{v}$ (below). The interface is colored according to its height: the lighter gray (white online) corresponds to higher region and the darker gray (blue online) corresponds to lower region. The dimension of the domain displayed here is $3 L_{x} \times 3 L_{y} \times L_{z}$. The aspect ratio is $L_{y} / L_{x}=0.5774 \approx 1 / \sqrt{3}$. Here, we show the result with the particle level-set method.

relation appears: $\boldsymbol{k}_{2}^{\prime}+\boldsymbol{k}_{2}=\boldsymbol{k}_{1}$, as shown in Fig. 6(c). This rhomboidal pattern involves two circles in the wavenumber space, which is a notable difference from the square and hexagonal patterns.

The experiments on the rhomboid patterns were reported in the two Refs. 9 and 10. There is a slight difference in the experimental settings between the references. We succeed in simulating the rhomboidal patterns with the same parameters for each of the two references. However, here we present only the result corresponding to the Ref. 9 since it contains a detailed analysis of the pattern along with a photograph of the rhomboidal pattern. Note that for the square and hexagonal patterns, we use the parameters of Ref. 10. The numerical parameters are listed in Table IV. We set the size of the horizontal domain again to be minimized containing one rhomboid, namely, $L_{x}=$ $2 \pi /\left(k_{1} / 2\right), L_{y}=2 \pi /\left(k_{2} \sin \varphi\right)$ where $\varphi=70.16$ is the angle between the vectors $\boldsymbol{k}_{1}$ and $\boldsymbol{k}_{2}$ shown in Fig. 6(c). It is calculated from the relation $2 k_{2} \cos \varphi=k_{1}$. The aspect ratio is thus $L_{y} / L_{x}=0.3620$. The numbers of grid points used per wavelength are $\left(2 \pi / k_{1}\right)\left(N_{x} / L_{x}\right)=0.5 N_{x},\left(2 \pi / k_{1}\right)\left(N_{y} / L_{y}\right)=$ $1.387 N_{y},\left(2 \pi / k_{2}\right)\left(N_{x} / L_{x}\right)=0.3408 N_{x}$, and $\left(2 \pi / k_{2}\right)\left(N_{y} / L_{y}\right)=0.9415 N_{y}$. The initial condition is set in the same way as the cases of the square and hexagonal patterns.

With the original level-set method, we do not obtain the rhomboidal state. On the other hand, with the particle level-set method, we obtain the state as a steady state as shown in Fig. 7. The first pattern 


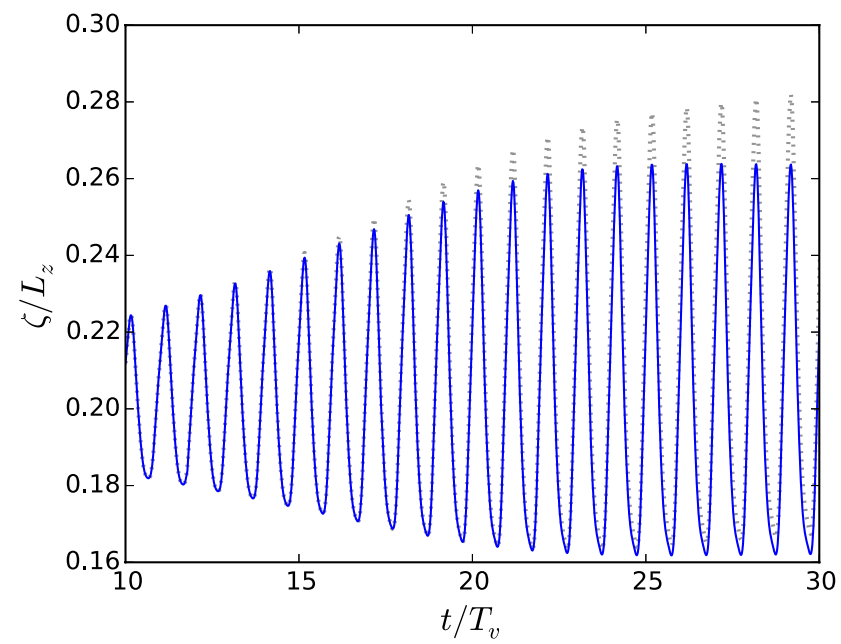

FIG. 5. Temporal variation of the interface height at position at $(x, y)=\left(0.5 L_{x}, 0.5 L_{y}\right)$ for the hexagonal pattern calculated with the particle level-set method (solid line) and with the original level-set method (dotted line).

recognition time of the rhomboid with the particle level-set method is $t \sim 29 T_{v}$ and the saturation time is the same $t \sim 29 T_{v}$ as depicted in Fig. 8. The first pattern recognition time is much longer than those of the square and hexagonal patterns. We consider that the nonlinear interaction among the resonant modes on the two circles in Fig. 6(c) takes a longer time in order to reach a constant oscillation amplitude.
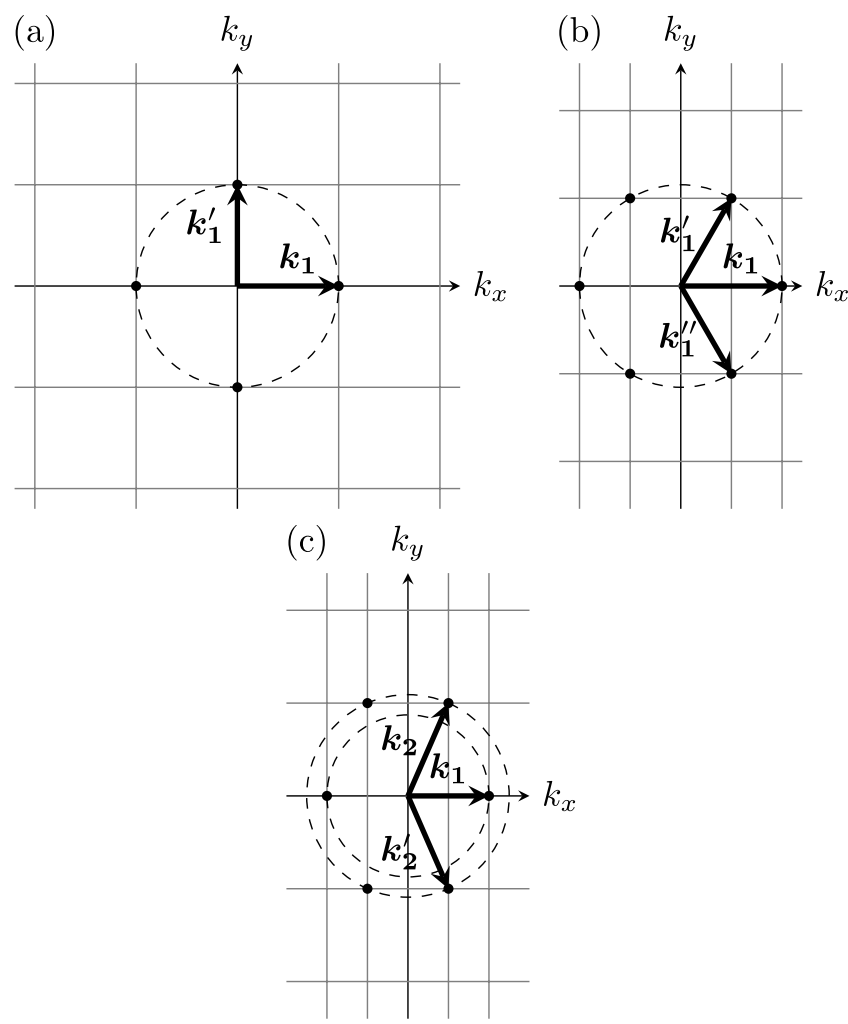

FIG. 6. Nonlinear resonant wavevectors (a) square pattern: $\left|\boldsymbol{k}_{1}\right|=\left|\boldsymbol{k}_{1}^{\prime}\right|=1.436 \times 10^{3}\left(\mathrm{~m}^{-1}\right)$. (b) Hexagonal pattern: $\left|\boldsymbol{k}_{1}\right|=\left|\boldsymbol{k}_{1}^{\prime}\right|=$ $\left|\boldsymbol{k}_{1}^{\prime \prime}\right|=1.061 \times 10^{3}\left(\mathrm{~m}^{-1}\right)$; the angle between $\boldsymbol{k}_{1}^{\prime}$ and the $k_{x}$ axis is $60^{\circ}$. (c) Rhomboidal pattern: $\left|\boldsymbol{k}_{1}\right|=8.653 \times 10^{2}\left(\mathrm{~m}^{-1}\right) ;\left|\boldsymbol{k}_{2}\right|=$ $\left|\boldsymbol{k}_{2}^{\prime}\right|=1.275 \times 10^{3}\left(\mathrm{~m}^{-1}\right)$; the angle $\varphi$ between $\boldsymbol{k}_{2}$ and the $k_{x}$ axis is $70.16^{\circ}$. The grid represents the minimal discretization of the Fourier space for each pattern. 
TABLE IV. Parameter values for the $2 k$ rhomboidal states. These parameters (except for $L_{x}, L_{y}, L_{z}, \theta, N_{x}, N_{y}$, and $N_{z}$ ) are identical with the experiment by Arbell and Fineberg. ${ }^{?}$

\begin{tabular}{lcc}
\hline \hline$L_{x}$ & $1.446 \times 10^{-2}$ & $(\mathrm{~m})$ \\
$L_{y}$ & $5.234 \times 10^{-3}$ & $(\mathrm{~m})$ \\
$L_{z}$ & $1.000 \times 10^{-2}$ & $(\mathrm{~m})$ \\
$\rho_{t}$ & 1.293 & $\left(\mathrm{kgm}^{-3}\right)$ \\
$\rho_{b}$ & $9.500 \times 10^{2}$ & $\left(\mathrm{kgm}^{-3}\right)$ \\
$\eta_{t}$ & $1.822 \times 10^{-5}$ & $\left(\mathrm{~kg} \mathrm{~m}^{-1} \mathrm{~s}^{-1}\right)$ \\
$\eta_{b}$ & $2.185 \times 10^{-2}$ & $\left(\mathrm{kgm}^{-1} \mathrm{~s}^{-1}\right)$ \\
$A_{1}$ & $2.372 \times 10^{1}$ & $\left(\mathrm{~ms}^{-2}\right)$ \\
$A_{2}$ & $4.925 \times 10^{1}$ & $\left(\mathrm{~ms}^{-2}\right)$ \\
$\omega_{1}=2 \omega_{0}$ & $3.141 \times 10^{2}$ & $\left(\mathrm{~s}^{-1}\right)$ \\
$\omega_{2}=3 \omega_{0}$ & $4.712 \times 10^{2}$ & $\left(\mathrm{~s}^{-1}\right)$ \\
$\theta$ & 0 & $(\mathrm{rad})$ \\
$\sigma$ & $2.150 \times 10^{-2}$ & $\left(\mathrm{kgm}^{-1}\right)$ \\
$g$ & 9.807 & $\left(\mathrm{~ms}^{-2}\right)$ \\
$N_{x} \times N_{y} \times N_{z}$ & $96 \times 32 \times 64$ & $(\mathrm{~m})$ \\
Bottom-fluid depth & $2.00 \times 10^{-3}$ & \\
\hline \hline
\end{tabular}

Figure 9 shows the temporal evolution of the Fourier amplitudes of the interface height for the three resonant modes. The circle symbols represent the simulation results, and the solid line is the evolution calculated with the Floquet coefficients obtained in the linear stability analysis. ${ }^{19}$ The evolution of the nonlinear rhomboidal modes (circle symbols in Fig. 9) is quite close to that of the linear results, which indicates that the nonlinear effect in the temporal evolution of the pattern is weak.

Now, we compare the simulation results with the weakly nonlinear analysis of the rhomboidal states by Porter and Silber. ${ }^{23,25}$ Their analysis for the first time explains with an elegant brokensymmetry argument why the rhomboidal pattern appears. In deriving their amplitude equations up to third order in the amplitude, they assume that the rhomboidal state is close to the bicritical point and that the damping parameter $\gamma$ is small. Accordingly, they expand the coefficients in the amplitude equations in powers of the vibration amplitudes $A_{1}^{*}=\left(A_{1}-A_{1 c}\right) / A_{1 c}$ and $A_{2}^{*}=\left(A_{2}-A_{2 c}\right) / A_{2 c}$ and the damping parameter $\gamma / \omega_{0}$. The resulting coefficients of the quadratic term of the amplitude, their signs, and dependence on $\gamma$, explain the rhomboidal pattern selection for certain frequency ratios $m: n$.

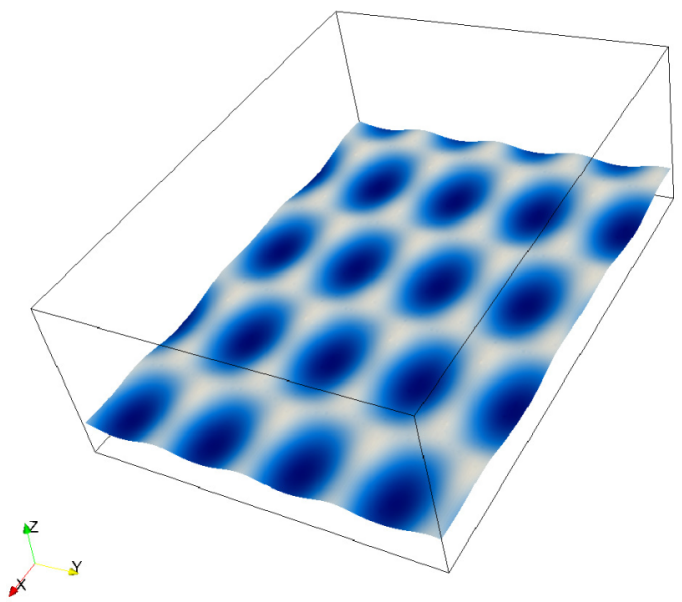

FIG. 7. Interface profile for the rhomboidal state at $t=40.38 T_{v}$. The interface is colored according to its height: the lighter gray (white online) corresponds to higher region and the darker gray (blue online) corresponds to lower region. The dimension of the domain displayed here is $2 L_{x} \times 4 L_{y} \times L_{z}$. The aspect ratio of the displayed domain is $4 L_{y} /\left(2 L_{x}\right)=0.7239$. Here we show the result with the particle level-set method. 


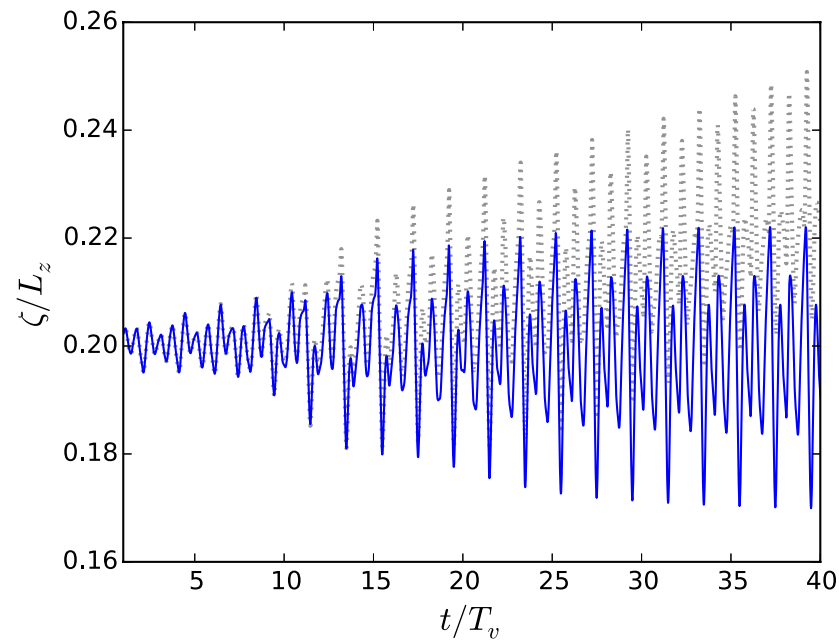

FIG. 8. Temporal variation of the interface height at position at $(x, y)=\left(0.3 L_{x}, 0.25 L_{y}\right)$ for the rhomboidal state calculated with the particle level-set method (solid line) and with the original level-set method (dotted line).

However, as they discussed, it is not clear that the damping parameter $\gamma / \omega_{0}$ is small enough in the experiments. ${ }^{9}$

To test the assumption, we measure the damping parameter from our simulation data. Before doing this, we calculate it with dimensional analysis: the damping parameter of the bottom fluid can be estimated as $\gamma(k) / \omega_{0}=2 \eta_{b} /\left(\rho_{b} k^{2} \omega_{0}\right)$ with the critical wavenumber $k$. This gives $\gamma\left(k_{1}\right) / \omega_{0}=$ 0.219 and $\gamma\left(k_{2}\right) / \omega_{0}=0.476$, where the critical wavenumbers $k_{1}$ and $k_{2}$ are determined for the critical vibration amplitudes $A_{1 c}=21.9$ and $A_{2 c}=44.8$. These dimensional values can differ in orders of magnitudes from the actual damping parameter. In our nonlinear simulation of the rhomboidal pattern, we set the normalized vibration amplitudes $A_{1}^{*}=(23.7-21.9) / 21.9=0.0831$ and

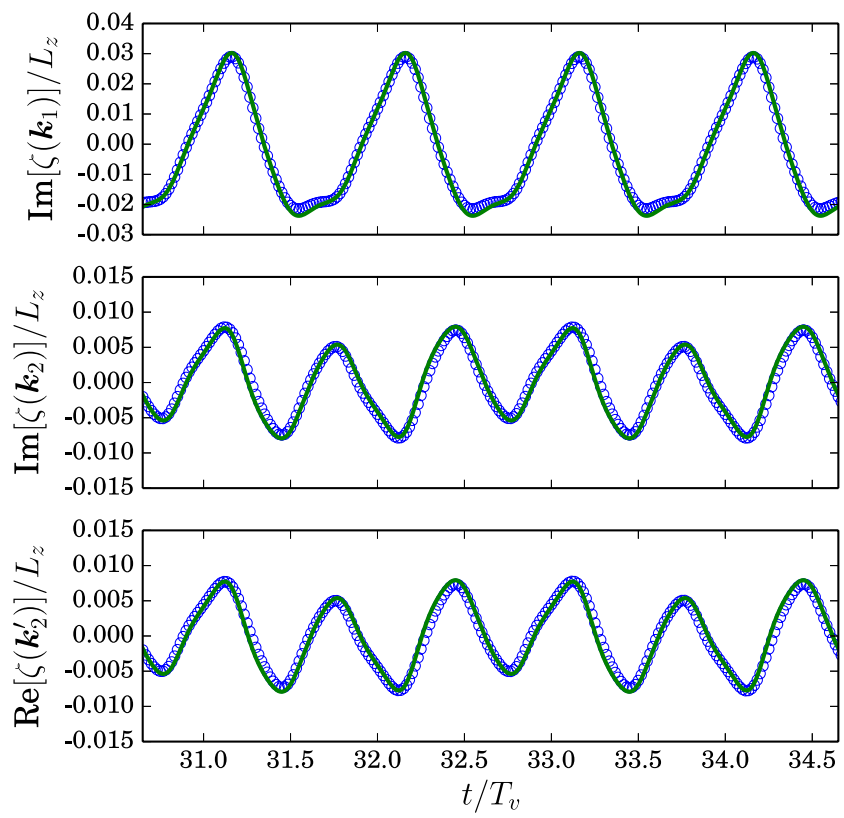

FIG. 9. Temporal evolution of the resonance amplitudes of the interface height $\zeta$ for the wavevectors $\boldsymbol{k}_{2}, \boldsymbol{k}_{2}^{\prime}, \boldsymbol{k}_{1}$ (see Fig. 6(c)) in the rhomboidal state. Here, we show only the dominant parts for each wavevector. The symbols are data of the simulation with the particle level-set method. The solid lines are time evolution of the neutral stable modes calculated with the ten Floquet coefficients in the linear stability analysis. 


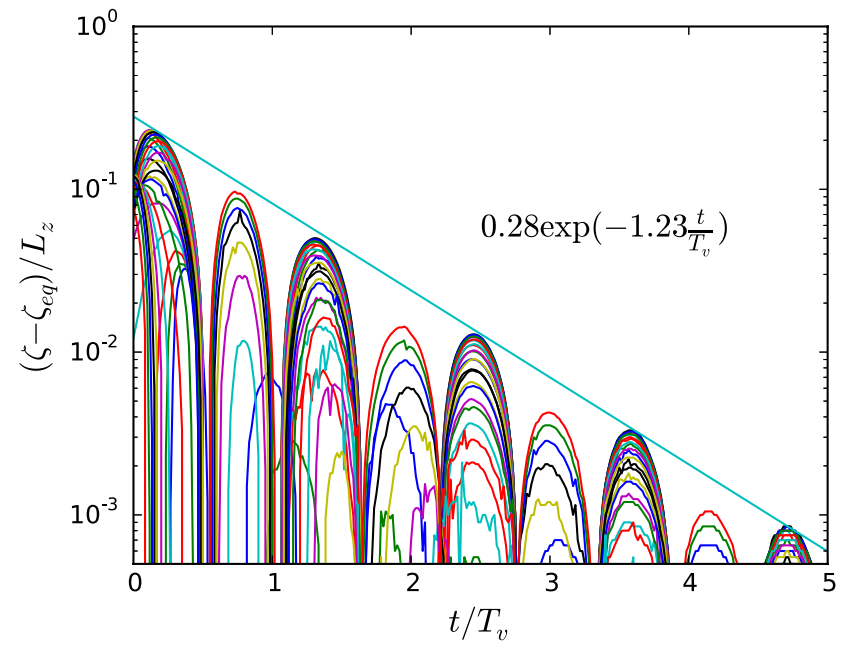

FIG. 10. Measurement of the damping parameter $\gamma$. The curves are temporal variations of the interface height at various points on the line $x=0$ without the vibration forcing. The damping parameter $\gamma / \omega_{0}$ can be estimated from the envelope $0.28 \exp \left(-1.23 t / T_{v}\right)$. The interface height at the quiescent state (where the Faraday waves decay completely) is denoted as $\zeta_{e q} / L_{z} \simeq 0.197$, which is slightly different from the initial interface height $0.20 L_{z}$ without the random perturbation.

$A_{2}^{*}=(49.3-44.8) / 44.8=0.100$, which justifies the expansion in terms of $A_{1}^{*}$ and $A_{2}^{*}$ in the coefficients of the weakly nonlinear analysis. In order to estimate the damping parameter in our nonlinear simulation, we used a method similar to that used in the experiment: ${ }^{38}$ we take the snapshot at $t=$ $141.4 T_{v}$ from the rhomboidal pattern simulation as an initial condition; we then start the simulation without the vibration forcing and measure how the interface elevation decays in time. The temporal interface behaviors on the line at $x / L_{x}=0$ are shown in Fig. 10. The envelope in the figure gives $\gamma T_{v} \sim 1.23$. Consequently, the damping parameter $\gamma / \omega_{0}$ is 0.196 . Although this is smaller than unity, it may not be small enough to ignore its higher order terms.

We next try to obtain the slowly varying amplitudes from the fully nonlinear evolution of the resonant modes shown in Fig. 9. For example, $\operatorname{Im} \zeta\left(\boldsymbol{k}_{2}\right)$ divided by the sub-harmonic oscillation $C \sin \left[2 \pi /\left(\omega_{2} / 2\right) t+\Theta\right]$, where $\omega_{2}=3 \omega_{0}, C$ is a suitable factor and $\Theta$ is a suitable phase, should give a slowly evolving function. We divided the resonant mode (symbols in Fig. 9) by the sub-harmonic oscillation. However, the calculated function is not slowly varying in time. Moreover, we divided the nonlinear data (symbols) by the linear Floquet-mode data (lines) in Fig. 9. The calculated function is not slowly varying either. Nevertheless, we look at the phase-space orbit formed by the three variables in Fig. 9. We do not find a characteristic structure often associated with the solutions of the normal-form equations corresponding to the rhomboidal structure. Hence, we are not able to compare our data with the weakly nonlinear analysis in this respect.

\section{Comparison between original and particle level-set method}

We observe that the original level-set method and the particle level-set method yield qualitatively different results. With the original level-set method, the square and hexagonal patterns are observed but do not become constant-amplitude oscillations. The rhomboidal state, which is here the main target, is not observed. On the other hand, in our simulation with the particle level-set method, all three patterns are observed and become constant-amplitude oscillations in agreement with the experiments. This difference is due to the well-known problem of the original level-set method, which we discuss here.

In order to clarify the difference between the two level-set methods, we look at how well the volume of the lower fluid is conserved during the time evolution. The volume of lower fluid is calculated with $H(\phi)$, Eq. (10), as $V(t)=\int H(\phi(x, t)) d \boldsymbol{x}$. The variations of the volume for the hexagonal 


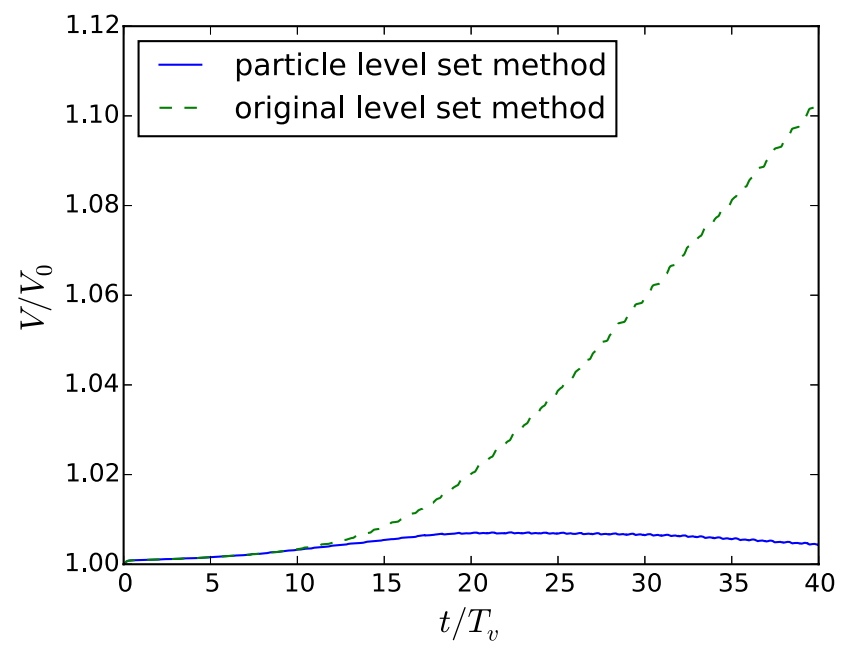

FIG. 11. Comparison of variation of the bottom fluid volume between the original level-set method and the particle level-set method in the case of the hexagonal pattern.

and rhomboidal cases are shown in Figs. 11 and 12 with the numerical parameters listed in Tables III and IV.

As shown in Figs. 11 and 12, the volume increases with the original level-set method, instead of being conserved. This non-conserving property of the original level-set method is well known. ${ }^{30-33}$ This explains why the interface height does not reach constant-amplitude oscillations with the original level-set method for the square and hexagonal patterns. Concerning the rhomboidal pattern, the original level-set method fails to exhibit the pattern. But with the particle level-set, we start to recognize rhomboids at $t=29 T_{v}$ (first recognition time). At this time, it is seen from Fig. 12 that the volume in the simulation with the original level-set method increases by $10 \%$. In other words, a long time is needed for the nonlinear interaction to form the resonant modes for the rhomboidal pattern. During this time, the error of the simulation with the original level-set method, the increase of the bottom-fluid volume, becomes so significant that the rhomboidal pattern is not observed. Therefore, we conclude that the particle level-set method is more suitable than the original level-set method to reproduce complex patterns such as the rhomboidal pattern, which require a long time for selection.

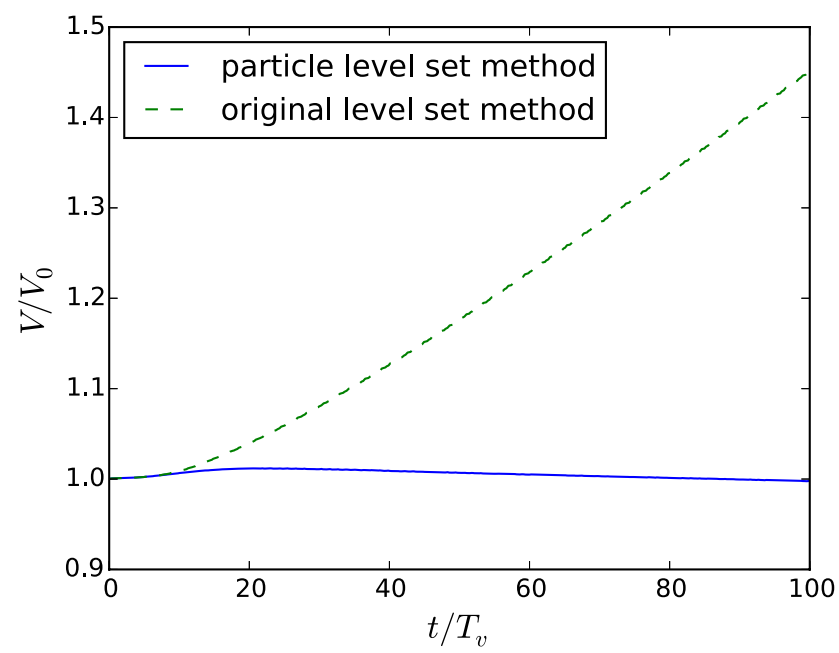

FIG. 12. Same as Fig. 11 but for the case of the rhomboidal states. 


\section{SUMMARY AND DISCUSSION}

Motivated by the recent experiments of Faraday waves with two or more frequency forcings exhibiting even richer patterns than the single frequency case, we have conducted a numerical simulation of the two-frequency Faraday waves, specifically targeting the rhomboidal pattern.

We first validated our numerical simulation with the linear stability analysis of the two-frequency Faraday waves. ${ }^{18}$ The two simple patterns, the square and hexagonal patterns, in the nonlinear regime were simulated with the same physical parameters as the experiment. ${ }^{10}$ In particular, the simulation using the particle level-set method in the minimal computational domain reproduced the two patterns in agreement with the experiment. Employing the particle level-set method, we finally reproduced numerically the $2 k$ rhomboidal states, the most complex pattern in this numerical study, with fluid properties identical to those of the experiments. We next checked whether the rhomboid obtained in our simulation satisfies the assumption made in the weakly nonlinear analysis for the rhomboidal pattern. ${ }^{23,25}$ Specifically, the assumption concerns the smallness of the damping parameter and the vibration amplitudes. We found that the damping parameter of the rhomboidal pattern in our simulation is marginally small. Further, comparison with the weakly nonlinear analysis is difficult.

In these simulations, we used two level-set methods: the original level-set method and the particle level-set method. The interface motion of the Faraday waves appears quite modest in the sense that it is not usually considered as a typical target of the interface-tracking schemes. One may think that any modern scheme is capable of simulating Faraday waves. However, due to the well-known problem of the original level-set method, ${ }^{32}$ we failed to simulate the square and hexagonal patterns as steady states and to reproduce the rhomboidal pattern at all. Thus, the Faraday wave problem requires an accurate scheme tracking of the interface such as the particle level-set method. One reason for this is that we need to simulate the system for a long time if we start with a random initial condition. We believe that, in developing a new implementation of the interface-tracking scheme, the Faraday wave problem can be a benchmark problem in addition to a physical phenomenon. In the linear regime, quantitative comparison can be made as demonstrated in the simulation by Périnet et al. ${ }^{16}$ In the nonlinear regime, qualitative comparison can be made (whether or not the right pattern emerges if we choose parameters for a certain pattern observed in experiments).

We carried out simulations on the minimal calculation domains to reproduce the three patterns. Its effect was studied for the rhomboidal case in the following way. Simulations were run in domains which were twice as large with twice as many points, thus keeping the density of numerical grid points constant. Accordingly, we have the same grid spacings in the physical space, $L_{x} / N_{x}$ and $L_{y} / N_{y}$ (for the $z$ direction, we keep the same values for $L_{z}$ and $N_{z}$ ). The rhomboidal pattern is observed with this setting with the same first pattern recognition time and the saturation time. Hence, it is unlikely that the minimal domain setting affects the pattern selection numerically. We also checked whether the number of grid points in the vertical direction $N_{z}$ is sufficient or not by doubling $N_{z}$ but retaining the other parameters as in Table IV. The result does not change.

As we mentioned briefly in the Introduction, many other patterns are observed in the experiments of the two-frequency forced Faraday waves. In fact, our initial goal was to reproduce not only the rhomboidal pattern but also the hexagonal based oscillon (HBO) (also known as DHS), the SSS and the oscillon observed experimentally by Arbell and Fineberg. ${ }^{9,10}$ So far, we have not been able to reproduce those patterns perhaps due to our strategy to use the minimal calculation domain including one pattern. Setting the minimal domain corresponds in terms of the Fourier space to maximizing the grid spacings in the $k_{x}$ and $k_{y}$ directions to include the resonant modes with discretized points. For these patterns, we failed to simulate; in fact, it is not clear how to set a minimal domain even with the knowledge of the selected resonant modes available from the experiments.

Now, we take the HBO pattern as an example and discuss the difficulty of setting the minimal domain. In the linear analysis of the HBO case, two different wavenumbers simultaneously become unstable. Hence, as in the case of the rhomboidal pattern shown in Fig. 6, two circles can be important. However, according to the experiment, the resonant modes lie on only one of the two, which we call the resonant circle; we call the other circle the non-resonant circle. As a first trial, we took the minimal calculation domain to resolve only these resonant modes on the resonant circle without including modes on the non-resonant circle. With this minimal domain and the particle level-set method, we 
did not obtain the HBO pattern at all starting from the same initial condition as in Sec. III. We speculate that, in the course of establishing the resonant modes, the modes on the non-resonant circle are important in the pattern selection and hence should be taken into account properly in the simulation. Of course, if we could enlarge the calculation domain and increase the number of grid points in the physical space in order to take a large number of mesh points near the non-resonant circle in the Fourier space, this problem might be overcome. Even though we have doubled $L_{x}, L_{y}, N_{x}$, and $N_{y}$, in order to take smaller grid spacing in the Fourier space, the HBO pattern did not emerge. A finer grid would make the cost of computation prohibitively high (notice that a long time simulation is also needed here).

We also ran the simulation of the SSS but failed possibly for the same reason. The resonant modes of the SSS observed experimentally lie either on a circle whose wavenumber (radius) is linearly stable or one of the two circles determined by the linear stability analysis. For both the HBO and the SSS cases, we checked that the volume is conserved to the same degree as it is in the rhomboidal case with the particle level-set method. Regarding the oscillon, the structure of the resonant modes in the Fourier space is not clarified experimentally, implying that we do not have any guidance on the discretization of the Fourier space. Perhaps, guessing from the physical-space appearance of the oscillon, the number of excited Fourier modes is very large compared with other patterns. To circumvent this sort of difficulty, a completely different numerical scheme with Chebychev polynomials for capturing a localized structure is proposed by Lloyd et al.,${ }^{39}$ which may be worth exploring. Moreover the oscillon's metastability ${ }^{10}$ may make simulation even more challenging. Our future work is an approach relying on computing power in which we take as high a resolution as possible to reproduce complex patterns like the HBO, the SSS, and the oscillon. We believe that if such a simulation succeeds, it would provide knowledge about the role of the modes on the non-resonant circles in pattern selection.

\section{ACKNOWLEDGMENTS}

This work is supported by the grant for JSPS fellows No. $25 \cdot 1056$ and by the JSPS KAKENHI (C) No. 25400400. We are grateful to Professor Sadayoshi Toh for his continuous encouragement. We thank anonymous referees for comments and for drawing our attention to the two Refs. 14 and 39.

${ }^{1}$ M. Faraday, "On a peculiar class of acoustical figures; and on certain forms assumed by groups of particles upon vibrating elastic surfaces," Philos. Trans. R. Soc. London 121, 299-340 (1831).

${ }^{2}$ F. Merkt, R. Deegan, D. Goldman, E. Rericha, and H. Swinney, "Persistent holes in a fluid," Phys. Rev. Lett. 92, 184501 (2004).

${ }^{3}$ G. Pucci, E. Fort, M. Ben Amar, and Y. Couder, "Mutual adaptation of a Faraday instability pattern with its flexible boundaries in floating fluid drops," Phys. Rev. Lett. 106, 024503 (2011)

${ }^{4}$ W. Edwards and S. Fauve, "Parametrically excited quasicrystalline surface waves," Phys. Rev. E 47, R788-R791 (1993).

${ }^{5}$ W. S. Edwards and S. Fauve, "Patterns and quasi-patterns in the Faraday experiment," J. Fluid Mech. 278, 123 (1994).

${ }^{6}$ H. Müller, "Periodic triangular patterns in the Faraday experiment," Phys. Rev. Lett. 71, 3287-3290 (1993).

${ }^{7}$ A. Kudrolli, B. Pier, and J. J. Gollub, "Superlattice patterns in surface waves," Phys. D 123, 99-111 (1998).

${ }^{8}$ H. Arbell and J. Fineberg, "Spatial and temporal dynamics of two interacting modes in parametrically driven surface waves," Phys. Rev. Lett. 81, 4384-4387 (1998).

${ }^{9}$ H. Arbell and J. Fineberg, "Two-mode rhomboidal states in driven surface waves," Phys. Rev. Lett. 84, 654-657 (2000)

${ }^{10}$ H. Arbell and J. Fineberg, "Temporally harmonic oscillons in Newtonian fluids," Phys. Rev. Lett. 85, 756-759 (2000).

${ }^{11} \mathrm{H}$. Arbell and J. Fineberg, "Pattern formation in two-frequency forced parametric waves," Phys. Rev. E 65, 036224 (2002)

12 T. Epstein and J. Fineberg, "Control of spatiotemporal disorder in parametrically excited surface waves," Phys. Rev. Lett. 92, 244502 (2004).

${ }^{13}$ T. Epstein and J. Fineberg, "Necessary conditions for mode interactions in parametrically excited waves," Phys. Rev. Lett. 100, 134101 (2008).

${ }^{14}$ C. Wagner, H.-W. Müller, and K. Knorr, "Pattern formation at the bicritical point of the Faraday instability," Phys. Rev. E 68, 066204 (2003).

${ }^{15}$ G. Tryggvason, R. Scardovelli, and S. Zaleski, Direct Numerical Simulations of GasLiquid Multiphase Flows (Cambridge University Press, 2011).

${ }^{16}$ N. Périnet, D. Juric, and L. S. Tuckerman, "Numerical simulation of Faraday waves,” J. Fluid Mech. 635, 1 (2009).

${ }^{17}$ A. Kityk, J. Embs, V. Mekhonoshin, and C. Wagner, "Spatiotemporal characterization of interfacial Faraday waves by means of a light absorption technique," Phys. Rev. E 72, 036209 (2005).

${ }^{18}$ T. Besson, W. Edwards, and L. Tuckerman, "Two-frequency parametric excitation of surface waves," Phys. Rev. E 54, 507-513 (1996).

${ }^{19}$ K. Kumar and L. S. Tuckerman, "Parametric instability of the interface between two fluids," J. Fluid Mech. 279, 49-68 (1994). 
${ }^{20}$ M. Silber and A. Skeldon, "Parametrically excited surface waves: Two-frequency forcing, normal form symmetries, and pattern selection,” Phys. Rev. E 59, 5446-5456 (1999).

${ }^{21}$ M. Silber, C. M. Topaz, and A. C. Skeldon, "Two-frequency forced Faraday waves: Weakly damped modes and pattern selection," Phys. D 143, 205-225 (2000).

${ }^{22}$ D. Tse, A. M. Rucklidge, R. Hoyle, and M. Silber, "Spatial period-multiplying instabilities of hexagonal Faraday waves," Phys. D 146, 367-387 (2000).

${ }^{23}$ J. Porter and M. Silber, "Broken symmetries and pattern formation in two-frequency forced Faraday waves," Phys. Rev. Lett. 89, 084501 (2002).

${ }^{24}$ C. M. Topaz and M. Silber, "Resonances and superlattice pattern stabilization in two-frequency forced Faraday waves," Phys. D 172, 1-29 (2002).

${ }^{25}$ J. Porter and M. Silber, "Resonant triad dynamics in weakly damped Faraday waves with two-frequency forcing," Phys. D 190, 93-114 (2004).

${ }^{26}$ C. Topaz, J. Porter, and M. Silber, “Multifrequency control of Faraday wave patterns," Phys. Rev. E 70, 066206 (2004).

${ }^{27} \mathrm{~W}$. Zhang and J. Viñals, "Pattern formation in weakly damped parametric surface waves driven by two frequency components," J. Fluid Mech. 341, 225-244 (1997).

${ }^{28}$ A. C. Skeldon and G. Guidoboni, "Pattern selection for Faraday waves in an incompressible viscous fluid," SIAM J. Appl. Math. 67, 1064-1100 (2007)

${ }^{29}$ M. Sussman, P. Smereka, and S. Osher, "A level set approach for computing solutions to incompressible two-phase flow," J. Comput. Phys. 114, 146-159 (1994).

${ }^{30}$ M. Sussman and E. Fatemi, "An efficient, interface-preserving level set redistancing algorithm and its application to interfacial incompressible fluid flow," SIAM J. Sci. Comput. 20, 1165-1191 (1999).

${ }^{31}$ D. Enright, R. Fedkiw, J. Ferziger, and I. Mitchell, "A hybrid particle level set method for improved interface capturing," J. Comput. Phys. 183, 83-116 (2002).

32 A. Prosperetti and G. Tryggvason, Computational Methods for Multiphase Flow (Cambridge University Press, 2009), p. 488.

${ }^{33}$ M. Sussman and E. G. Puckett, "A coupled level set and volume-of-fluid method for computing 3D and axisymmetric incompressible two-phase flows," J. Comput. Phys. 162, 301-337 (2000).

${ }^{34}$ G.-S. Jiang and D. Peng, "Weighted ENO schemes for Hamilton-Jacobi equations," SIAM J. Sci. Comput. 21, 2126-2143 (2000).

${ }^{35}$ H. Takahira, T. Horiuchi, and S. Banerjee, "An improved three-dimensional level set method for gas-liquid two-phase flows," J. Fluids Eng. 126, 578 (2004).

${ }^{36}$ Y. Saad, Iterative Methods for Sparse Linear Systems, 2nd ed. (SIAM, 2003).

${ }^{37}$ N. Périnet, D. Juric, and L. S. Tuckerman, "Alternating hexagonal and striped patterns in Faraday surface waves," Phys. Rev. Lett. 109, 164501 (2012)

${ }^{38}$ B. Cocciaro, S. Faetti, and M. Nobili, "Capillarity effects on surface gravity waves in a cylindrical container: Wetting boundary conditions," J. Fluid Mech. 231, 325 (1991).

${ }^{39}$ D. J. B. Lloyd and A. R. Champneys, "Efficient numerical continuation and stability analysis of spatiotemporal quadratic optical solitons," SIAM J. Sci. Comput. 27, 759-773 (2005). 